\title{
Screening and diagnosis of chronic HCV infection in Bulgaria: a review of the current practice.
}

\author{
Maria Dimitrova $^{1 *}$, Maria Kamusheva ${ }^{1}$, Konstantin Mitov ${ }^{1}$, Daniela Grekova ${ }^{2}$, Guenka Petrova $^{1}$ \\ ${ }^{1}$ Medical University of Sofia, Sofia, Bulgaria \\ ${ }^{2}$ Medical University of Plovdiv, Plovdiv, Bulgaria
}

\begin{abstract}
Background: Chronic HCV infection is one of the leading causes of chronic liver disease and places significant health and economic burden worldwide. In Bulgaria blood and blood products weren`t screened before 1992. Currently there is no official national screening program and diagnosis is usually accidental and in more advanced stages of the disease.

Methods and materials: A synthetic, population-based decision tree with Markov chain model compares the current practice (No screening) with possible screening program among (Screening) the people aged 39-64 as a part of the routine prophylaxis program. A Markov chain model was built at the end every decision node of the patients without Sustained Viral Response (SVR) to find how they will progress for the observed period.

Results: Possible national screening would lead to almost 4 times higher number of patients diagnosed at early stages of the infection, almost 2 to 4 times higher number of patients with access to treatment and decrease in the HCV-related expected mortality. Expected life years gained in the Screening alternative are almost 2 (10 417 LYGs) and 4 times (22 715 LYGs) higher compared to the current situation (4798 LYGs), respectively. Only $45 \%$ of the people however perform routine prophylaxis.

Conclusion: National screening is considered as effective health care measure to fight chronic diseases because through active search we can find asymptomatic patients. Initiation of treatment in early stages of hepatitis $\mathrm{C}$ infection is more effective and decreases the mortality rates.
\end{abstract}

Keywords: Chronic HCV infection, Screening, Birth-cohort, Eradication.

Accepted on June 25, 2018

\section{Introduction}

Chronic HCV infection is a significant health and economic burden worldwide, as it is one of the leading causes of chronic liver disease and related complications as extensive fibrosis, cirrhosis and Hepatocellular Carcinoma (HCC) [1,2]. World Health Organization (WHO) data show that 71 million people worldwide are diagnosed with chronic HCV infection and 399 000 are dying every year because of HCV-related cirrhosis and HCC. Highest prevalence rates are observed in the Eastern Mediterranean and the Eastern European WHO Regions-2.3\% and $1.5 \%$, respectively. [3].

Late diagnosis of the chronic HCV infection is related not only to increased risk of manifestation of the long-term complications, cirrhosis, HCC, and premature mortality but also to higher economic burden as well. Studies have shown that the cost of treatment has a positive correlation with the progression of the disease [4].

The acute HCV and chronic infections are usually asymptomatic that leads to late diagnosis after the clinical manifestation of the symptoms related to progressed liver disease [3].

The standard of treatment has changed significantly in the recent years with the authorization of the new interferon-free regimes with Direct Acting Agents (DAAs). The therapeutic success increased significantly to $95 \%-97 \%$ Sustained Viral Response (SVR) depending on the fibrosis stage and cirrhosis [5-9]. Therefore a necessity of earlier diagnosis became indubitable to prevent the future complications and deaths.

The global health sector strategy of WHO aims to increase the percentage of diagnosed patients from less than $5 \%$ in 2015 ; to $30 \%$ in 2020; and $90 \%$ in 2030 for viral hepatitis B and C. This is expecting to result in higher percentage of earlier diagnosed patients with access to treatment. Most of these strategies could be achieved with the implementation of national screening programs for early diagnosis of chronic viral hepatitis infections [10]. Studies have shown that initiation of treatment before the age of 50 in early fibrosis stages $\left(\mathrm{F}_{0}-\mathrm{F}_{2}\right)$ leads to 13 life years gained (LYG) and to 7 LYG for later stages (F3-F4) [11]. 
WHO recommends three major screening methods based on the prevalence of the virus in the general population as focused testing in most affected populations, general population testing, and "birth-cohort" testing. "Birth cohort" testing is applicable to specific identified birth cohorts of older persons at higher risk of infection (i.e. historical exposure to unscreened or inadequately screened blood products and/or poor injection safety) and morbidity within populations that have an overall lower general prevalence [12].

The prevalence of HCV in the Bulgarian population according to expert opinion is approximately $1.1 \%$ and out of them $87 \%$ of the patients is expected to progress to chronic infection $[13,14]$. The most-at-risk group with highest prevalence rate according to experts are the persons above the age of 45 because the blood and blood products were screened after 1992 [15].

Currently there is no official national population screening program for HCV. Experts` data show that more than $50 \%$ of the patients are diagnosed at moderate-to-severe stages of the disease [16].

This prompted our interest towards the best possible option for earlier detection of HCV in Bulgaria. The goal of this study is to evaluate the current testing and diagnostic practice of chronic hepatitis $\mathrm{C}$ in Bulgaria and the future possibilities for implementation of a national screening program. We wanted to explore the possibility for Bulgaria to achieve the WHO target goals by implementing the recommended scenarios for $\mathrm{HCV}$ screening through constructing different screening scenarios. The point of view of the analysis is that of society for 12 years' time horizon.

\section{Methods and Materials}

A synthetic, population-based decision tree with Markov chain model was built gathering local epidemiology data and expert opinion for prevalence of $\mathrm{HCV}$ and progression of the disease at time of diagnosis. Data for the prevalence of $\mathrm{HCV}$ and progression of the disease at time of diagnosis were taken from the pharmaco-therapeutic guideline of gastrointestinal disorders, expert opinion and published epidemiology studies. Target population was identified from the official database of the National Statistics Institute (NSI) for the period 2018-2030 (Table 1).

The decision tree model has two major branches. The first one is No Screening alternative for people that could be screened and found during the current practice of HCV testing and diagnosis practice. The second branch is the Screening alternative in case of national screening program (Figures 1-3). Time horizon of 12 years (2019-2030) was considered. The model was built with the following assumptions:

(1) Population at risk for the Screening alternatives are all people at the age group 39-64, because no program for screening of blood and blood products existed before 1992 . Those patients will be catches up during routine health prophylaxis performed on every 5 years.
(2) In the Screening alternative the target population with anti$\mathrm{HCV}$ antibodies is proportionally distributed in age groups for the proposed period;

(3) In the Screening alternative the target population would be screened for anti-HCV antibodies only once in the lifetime left;

(4) In the No Screening alternative only $2 \%$ of the people with chronic HCV infection will be diagnosed, according to expert opinion data for the current diagnosis rate and the fact that in the mild stages the disease is asymptomatic and the patients aren't aware of the disease.

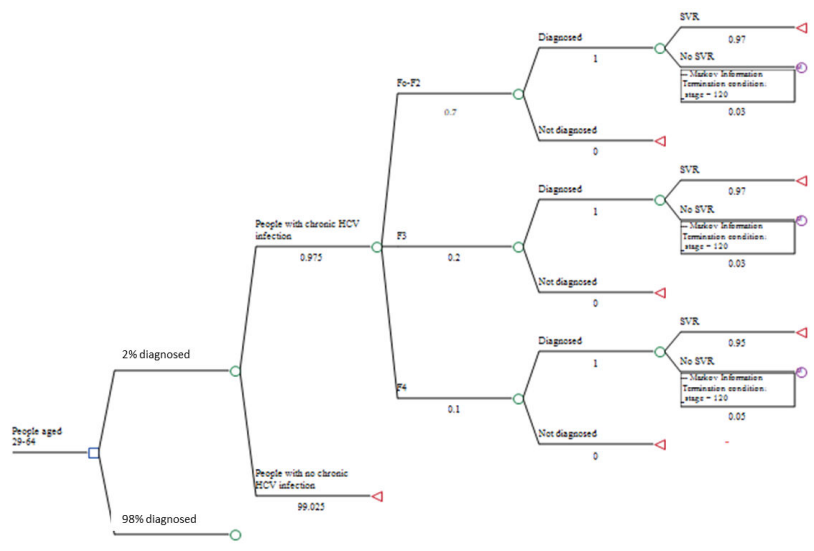

Figure 1. Decision tree model for scenario 1-no screening (Current practice on testing and diagnosis of $\mathrm{HCV}$ infection).

Based on these assumptions three decision tree scenarios were developed:

(1) No screening (current practice)-no screening in the target population is done and only $2 \%$ of the people are diagnosed occasionally (Figure 1).

(2) Active screening-all people in the target group are screened for HCV infection once in life time starting at 2019 (Figure 2).

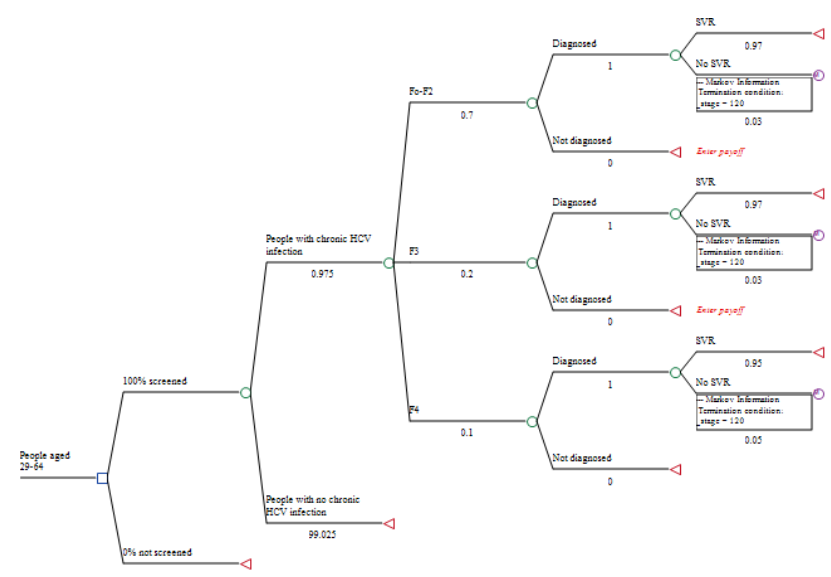

Figure 2. Decision tree model for scenario 2-active screening.

(3) Passive screening-people at age group 39-64 who go to annual obligatory routine prophylaxis are directed by the general practitioners for screening for $\mathrm{HCV}$ once in life time starting at 2019 (Figure 3). 


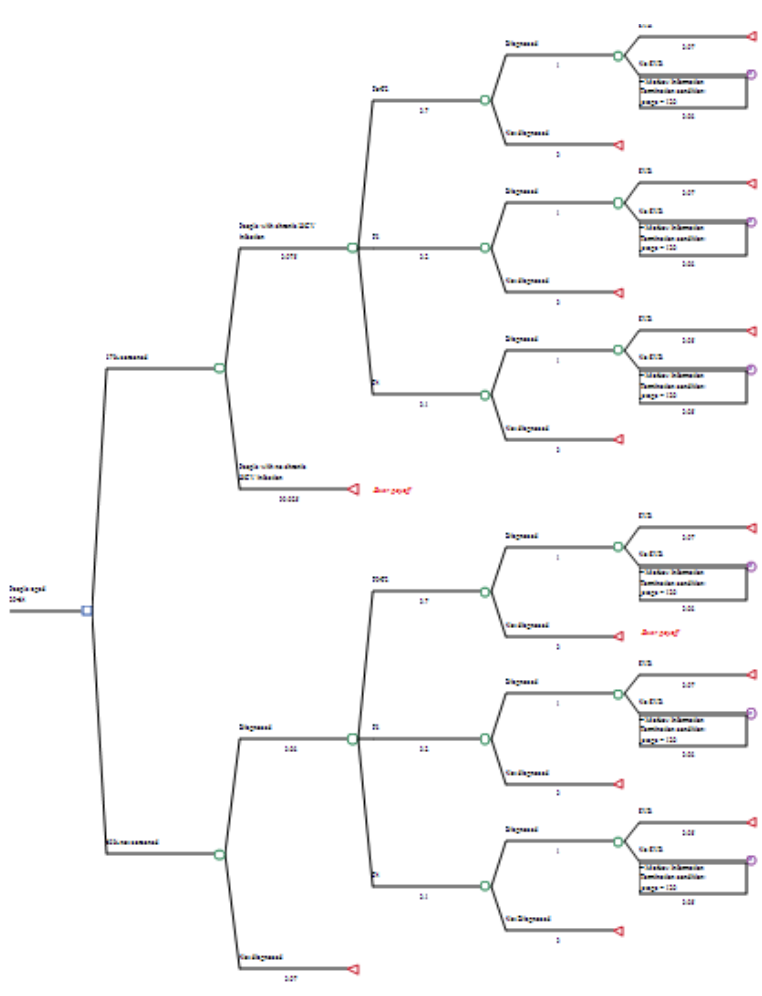

Figure 3. Decision tree model for scenario 3-passive screening during prophylactic examinations.

The probabilities used in the models are presented in Table 2.

The decision tree models for all scenarios are presented on Figures 1-3.

For all patients who remain undiagnosed from the No Screening and Passive Screening Scenario and for all patients who fail the treatment and remain uneradicated from the virus a Markov chain model (Figure 4) was built at the end every decision node of the patients without Sustain Viral Response (SVR) to find how they will progress for the observed period.

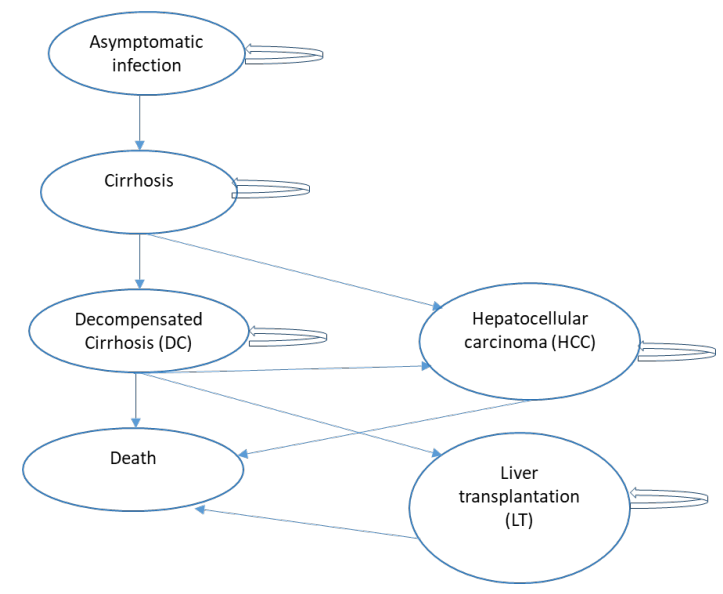

Figure 4. Bubble diagram for the Markov model.

The annual probabilities for progression from one state to other in the Markov model are presented in Table 3.
The transition probabilities in the Markov model were calculated by using the following formula for transforming the annual probabilities.

$T p=1-1 / e^{\mathrm{p}}$,

where $t^{\mathrm{p}}$ is the transitory probability, and $p$-annual probability for each health state

Life Years Gained (LYG) due to earlier diagnosis, therapy and prevented deaths were considered as a measure of effectiveness of the accepted screening scenario. Total LYGs for the three scenarios were calculated as the total number of early diagnosed patients with achieved SVR was calculated with the multiplied with the expected life years obtained when starting therapy before the age of 50 according to the study conducted by Maor et al. [11-13] LYs obtained in $\mathrm{F}_{0}-\mathrm{F}_{2}$ and 7 LYs-in $\mathrm{F}_{3}$ and $\mathrm{F}_{4}$, respectively.

Total LYGs $\left(\mathrm{F}_{0}-\mathrm{F}_{2}\right)=$ Total number of diagnosed and successfully treated $\mathrm{F}_{0}-\mathrm{F}_{2}$ patients $\times 13$ LYGs

Total LYGs $\mathrm{F}_{3}=$ total number of diagnosed and successfully treated $\mathrm{F}_{3}$ patients $\times 7 \mathrm{LYs}$

Total LYGs $\mathrm{F}_{4}=$ total number of diagnosed and successfully treated $\mathrm{F}_{4}$ patients $\times 7 \mathrm{LYs}$

\section{Results}

\section{Target population: no screening scenario}

Currently people are diagnosed with chronic HCV infection accidentally (personal decision, doctor's concern, blood donation, surgical interventions, etc.) or when the clinical symptoms are already manifesting-in stages $F_{3}$ and $F_{4}$ [16]. Expert opinion shows that in Bulgaria only $30 \%$ of the patients currently are diagnosed at $\mathrm{F}_{0}-\mathrm{F}_{2}$ stages, mostly between the age of 29 and 64 (Appendix 1).

Applying the decision tree approach for the first scenario (No screening-current practice) lead us to 565 persons diagnosed annually with chronic HCV infection, from which patients with cirrhosis would be on average 108, 5 with decompensated cirrhosis, 8 with HCC and 3 would be indicated for liver transplantation. Approximately 27,400 people with chronic HCV infection will remain undiagnosed. The total number of LYG will be 4798 (Table 4).

\section{Target population: screening scenarios}

The screening scenarios are based on data for the prevalence of $\mathrm{HCV}$ in the general population according to WHO recommendations (Appendix 2). Passive screening scenario is based on the "birth cohort method" given the assumptions explained in the methodology. Detailed distribution of patients is presented on Appendix 4. The passive screening scenario leads us 965 people diagnosed yearly due to recommendation for testing from the physicians during their prophylactic examination. Out of them in fibrosis stage $F_{3}$ will be 192 patients, in stage 4 will be 101 patients, 96 will eradicate the infection is proper therapy is applied, and 1159 will remain 
undiagnosed. The total number of LYG in this scenario will be 10417.

If active screening is performed in the target group and considering that $90 \%$ of the population in Bulgaria is health insured on average 218746 people aged 39-64 should be tested on yearly basis (Appendix 3). This would help to find on average 1465 patients in $\mathrm{F}_{0}-\mathrm{F}_{2}$ stage. This will increase the diagnosis at early stages from $30 \%$ to $70 \%$. For the observed period we expect to diagnose on total 25,121 patients with chronic HCV infection, from which 24,317 are expected to be cured, and to gain 22715 lives (Table 4).

As a result after solving the 3 created scenarios and the assumptions made a possible implementation of a testing for
HCV infection as a part of the routine prophylaxis program among the people at risk would result in almost 4 to 8 times higher number of patients diagnosed at early stages of the infection and almost 2 to 4 times higher number of patients with access to treatment. Expected life years gained in the Passive Screening and the Active Screening are almost 2 (10,417 LYGs) and 4 times (22,715 LYGs) higher compared to the current situation (4798 LYGs), respectively (Table 4).

After applying the Markov model for patients who do not achieve SVR in the 3 created scenarios and comparing with the current practice we found that the screening will result in decrease in the expected HCV-related mortality (Table 5).

Table 1. Input data in the population model.

\begin{tabular}{|c|c|c|}
\hline Indicator & Value & Source \\
\hline Total population & $7,101,859$ & NSI [17] (data for 2016) \\
\hline Average life expectancy & 75 y & NSI [17] (data for 2016) \\
\hline Population aged 29-64 (for No Screening scenario) & 3504141 & NSI [17] (data for 2016) \\
\hline Population aged 39-64 (for the Screening Scenario) & 2502397 & \\
\hline$\%$ of health insured people & $90 \%$ & NSI [17] \\
\hline No of health insured people aged 39-64 & 2252157 & NSI [17] (data for 2016) \\
\hline Annual number of people with obligatory routine prophylaxis & 225216 & Regulation 8/03.11.2016 [18] \\
\hline Percentage of people who go to routine health prophylaxis & $45 \%$ & NHIF [19] \\
\hline $\mathrm{HCV}$ prevalence in the general population & $1.10 \%$ & $\begin{array}{l}\text { Guideline on Gastrointestinal disorders [13], Antonov } \\
\mathrm{KA}[14]\end{array}$ \\
\hline$\%$ of patients with chronic $\mathrm{HCV}$ infection out of $1.1 \%$ infected & $87 \%$ & {$[16]$} \\
\hline Prevalence of the chronic $\mathrm{HCV}$ in the general population & $0.98 \%$ & {$[16]$} \\
\hline$\%$ of accidently diagnosed currently & $2 \%$ & Expert opinion \\
\hline $\begin{array}{l}\text { Average life years gained (LYG) when initiating antiviral therapy in stages } F_{0}-F_{2} \text { before the } \\
\text { age of } 50\end{array}$ & 13 & {$[11]$} \\
\hline $\begin{array}{l}\text { Average life years gained (LYG) when initiating antiviral therapy in stages } F_{3} \text { and } F_{4} \text {, } \\
\text { respectively before the age of } 50\end{array}$ & 7 & {$[11]$} \\
\hline
\end{tabular}

Table 2. Probabilities in the decision tree model.

\begin{tabular}{lll}
\hline Indicator & Value & Source \\
\hline Screening alternative & & \\
\hline Patients with mild chronic HCV infection $\left(F_{0}-F_{1}\right)$ & $50 \%$ & {$[16]$} \\
\hline Patients with moderate-severe chronic HCV infection: & $50 \%$ & {$[16]$} \\
\hline$F_{2}$ & $20 \%$ & \\
\hline$F_{3}$ & $20 \%$ & \\
\hline$F_{4}$ & $10 \%$ & \\
\hline No screening alternative & & \\
\hline $\begin{array}{l}\text { Patients with mild to moderate chronic HCV infection } \\
\left(F_{0}-F_{2}\right)\end{array}$ & $30 \%$ & Expert \\
\end{tabular}

\begin{tabular}{lll}
\hline $\begin{array}{l}\text { Patients with moderate to severe chronic HCV infection } \\
\mathrm{F}_{3}\end{array}$ & $40 \%$ & $\begin{array}{l}\text { Expert } \\
\text { opinion }\end{array}$ \\
\hline $\begin{array}{l}\text { Patients with moderate to severe chronic HCV infection } \\
\mathrm{F}_{4}\end{array}$ & $30 \%$ & $\begin{array}{l}\text { Expert } \\
\text { opinion }\end{array}$ \\
\hline$\%$ of patients with SVR $\mathrm{F}_{0}-\mathrm{F}_{3}$ & $97 \%$ & {$[5-8]$} \\
\hline$\%$ of patients with SVR $\mathrm{F}_{4}$ & $95 \%$ & {$[5-8]$} \\
\hline
\end{tabular}

Table 3. Annual probabilities in the Markov model.

\begin{tabular}{lll}
\hline Indicator & Value & Source \\
\hline Asymptomatic chronic infection->cirrhosis (F4) & 0.1 & {$[20]$} \\
\hline Cirrhosis->decompensated cirrhosis & 0.04 & {$[19]$} \\
\hline Cirrhosis->death & 0.05 & {$[19]$} \\
\hline
\end{tabular}


Screening and diagnosis of chronic HCV infection in Bulgaria: a review of the current practice

\begin{tabular}{lcc}
\hline Decompensated cirrhosis->HCC & 0.03 & {$[19]$} \\
\hline Decompensated cirrhosis->LT & 0.01 & {$[19]$} \\
\hline Decompensated cirrhosis->death & 0.05 & {$[19]$}
\end{tabular}

\begin{tabular}{lll}
\hline HCC->death & 0.05 & {$[19]$} \\
\hline LT ->death & 0.05 & {$[19]$} \\
\hline
\end{tabular}

Table 4. Average number of patients with access to therapy and successful treatment.

\begin{tabular}{|c|c|c|c|}
\hline \multirow[t]{2}{*}{ Indicator } & \multicolumn{3}{|l|}{ Alternative } \\
\hline & No screening & Active screening & Passive screening \\
\hline Population screened & 0 & 162173 & 98436 \\
\hline Patients found with $F_{0}-F_{2}$ & 169 & 1465 & 666 \\
\hline $\mathrm{F}_{0}-\mathrm{F}_{2}$ patients with virus eradication & 164 & 1421 & 646 \\
\hline Patients found $F_{3}$ & 226 & 419 & 198 \\
\hline F3 patients with virus eradication & 219 & 407 & 192 \\
\hline Patients found $F_{4}$ & 169 & 210 & 101 \\
\hline F4 patients with virus eradication & 161 & 199 & 96 \\
\hline Avg annual number of non-diagnosed patients & 27678 & 0 & 1128 \\
\hline Avg annual number of found patients with access to therapy & 565 & 2095 & 965 \\
\hline Avg annual number of patients virus eradication & 545 & 2028 & 934 \\
\hline Avg annual number of patients with no virus eradication & 27698 & 67 & 1159 \\
\hline Total number of people with virus eradication for the observed period & 6534 & 24317 & 11209 \\
\hline LYG & 4798 & 22715 & 10417 \\
\hline
\end{tabular}

Table 5. Markov model of the progression of the patients for the observed period.

\begin{tabular}{|c|c|c|c|c|c|c|}
\hline \multicolumn{7}{|c|}{ No of patients in the different health states for a 12 year period } \\
\hline \multirow[t]{2}{*}{ Year } & \multicolumn{6}{|c|}{ Scenario 1: No screening } \\
\hline & Inf & cirrhosis & DC & $\mathrm{HCC}$ & LT & Death \\
\hline 0 & 27698 & 0 & 0 & 0 & 0 & 0 \\
\hline 1 & 25061 & 2637 & 0 & 0 & 0 & 0 \\
\hline 2 & 22674 & 4791 & 103 & 0 & 0 & 129 \\
\hline 3 & 20515 & 6529 & 282 & 3 & 1 & 368 \\
\hline 4 & 18562 & 7907 & 514 & 11 & 4 & 700 \\
\hline 5 & 16794 & 8979 & 778 & 26 & 9 & 1112 \\
\hline 6 & 15195 & 9787 & 1062 & 48 & 16 & 1590 \\
\hline 7 & 13748 & 10373 & 1352 & 77 & 26 & 2122 \\
\hline 8 & 12439 & 10769 & 1640 & 113 & 38 & 2699 \\
\hline 9 & 11255 & 11005 & 1917 & 156 & 53 & 3312 \\
\hline 10 & 10183 & 11108 & 2180 & 205 & 69 & 3953 \\
\hline 11 & 9213 & 11100 & 2423 & 260 & 87 & 4615 \\
\hline Year & \multicolumn{6}{|c|}{ Scenario 2: Active screening } \\
\hline 0 & 67 & 0 & 0 & 0 & 0 & 0 \\
\hline
\end{tabular}

\begin{tabular}{|c|c|c|c|c|c|c|}
\hline 1 & 61 & 6 & 0 & 0 & 0 & 0 \\
\hline 2 & 55 & 12 & 0 & 0 & 0 & 0 \\
\hline 3 & 50 & 16 & 1 & 0 & 0 & 1 \\
\hline 4 & 45 & 19 & 1 & 0 & 0 & 2 \\
\hline 5 & 41 & 22 & 2 & 0 & 0 & 3 \\
\hline 6 & 37 & 24 & 3 & 0 & 0 & 4 \\
\hline 7 & 33 & 25 & 3 & 0 & 0 & 5 \\
\hline 8 & 30 & 26 & 4 & 0 & 0 & 7 \\
\hline 9 & 27 & 27 & 5 & 0 & 0 & 8 \\
\hline 10 & 25 & 27 & 5 & 0 & 0 & 10 \\
\hline 11 & 22 & 27 & 6 & 1 & 0 & 11 \\
\hline Year & Scena & Pass & & & & \\
\hline 0 & 1159 & 0 & 0 & 0 & 0 & 0 \\
\hline 1 & 1049 & 110 & 0 & 0 & 0 & 0 \\
\hline 2 & 949 & 201 & 4 & 0 & 0 & 5 \\
\hline 3 & 859 & 273 & 12 & 0 & 0 & 15 \\
\hline 4 & 777 & 331 & 21 & 0 & 0 & 29 \\
\hline 5 & 703 & 376 & 33 & 1 & 0 & 47 \\
\hline 6 & 636 & 410 & 44 & 2 & 1 & 67 \\
\hline
\end{tabular}




\begin{tabular}{lllllll}
\hline 7 & 575 & 434 & 57 & 3 & 1 & 89 \\
\hline 8 & 521 & 451 & 69 & 5 & 2 & 113 \\
\hline 9 & 471 & 461 & 80 & 7 & 2 & 139 \\
\hline 10 & 426 & 465 & 91 & 9 & 3 & 165 \\
\hline 11 & 386 & 465 & 101 & 11 & 4 & 193 \\
\hline
\end{tabular}

\section{Discussion}

National screening is considered as effective health care measure to fight chronic diseases because through active search we can find asymptomatic patients. Initiation of treatment in early stages of hepatitis $\mathrm{C}$ infection, respect is more effective and decreases the mortality rates [21].

The development of new therapies for chronic HCV infection with high cure rates $(90 \%-100 \%$ SVR at $12 \mathrm{w})$ and no major side effects led to changes in many public health programs towards early identifying of $\mathrm{HCV}$ in the population at risk [22]. Agencies like National Institute for Health and Care Excellence (NICE) and the Public Health Agency of Canada initiated studies to evaluate the cost-effectiveness of implementation of HCV screening programs [23]. They found that the national screening program would help to find the undiagnosed patients among the population at risk and to ensure timely access to treatment, respective to their genotype [24].

Studies have shown that if patients are found and treated before cirrhotic impairment they would have life expectancy similar to that of uninfected people [25]. If the patients progress to cirrhosis before the treatment initiation, viral eradication would eliminate the risk of liver failure and would significantly reduce the risk of hepatocellular carcinoma [26].

In this light not surprisingly, screening for HCV became one of the major strategic aims of WHO to decrease the morbidity and mortality of chronic HCV worldwide [11,27].

A Canadian study on the benefits and risks of screening of $\mathrm{HCV}$ showed that if birth cohort screening is implemented for people born $1945-1975$, up to $77 \%$ of the infections would be captured. The potential risks associated with the implementation of a screening program were the social stigma towards those infected with chronic $\mathrm{HCV}$ infection, false positive results and lack of capacity to treat all newly diagnosed cases [28].

Study, conducted in the European Union, shows that there is a need to develop targeted programmes for detection of $\mathrm{HCV}$ and other infections leading to chronic liver disease. The authors recommend public health programmes to be made according to local epidemiological data, new research evidence on efficiency and effectiveness and the innovations in the health system [29].

The Bulgarian pharmacotherapeutic guideline on gastrointestinal diseases recommends screening among population at increased risk according to the epidemiological patterns in the country but to date there is no official national screening program for $\mathrm{HCV}$ infection in Bulgaria.

The results from our study show that if screening scenario of the „birth cohort" is implemented in Bulgaria we could increase with almost $30 \%$ the number of diagnosed people with asymptomatic chronic $\mathrm{HCV}$ infection. If scenario active screening is applied we will have better opportunities to find and treat more patients in comparison with the passive screening scenario. Active screening reaches almost two times higher number of patients diagnosed and cured in early stages and LYGs. Further analysis should be done by applying costing procedures to the three possible scenarios to determine the cost-effective one.

It should be noted that our study possesses some limitations. The model is based on assumptions from published epidemiological data due to the lack of national epidemiology data, especially for the diagnosis of patients at asymptomatic stages of the infection which currently happens mostly by accident (blood donor testing, surgical interventions, personal decision).

It also focuses only on chronic HCV infection which is not the only reason for cirrhosis, hepatocellular carcinoma and liver transplantation in Bulgaria so their effect might be overestimated. This model cannot also meet the criteria of WHO for eradication of HCV until 2030 as it does not include the high-risk groups as (sexual workers, drug-abusers, etc.) for which there are some special programs for diagnosis and treatment. This might be considered as a limitation but our study wanted to focus on that part of the population which is not included in the latter groups but is still considered as a risk population and for which currently diagnosis is accidental and usually at late stages.

According to the experts chronic HBV infection affects 3\% of the total population and is other major cause of chronic liver disease with its complications [13]. Study, published in 2011, shows that the universal vaccination against HBV is socially beneficial for the Bulgarian population and is having positive economic effects that became visible during the latest 10 years since its introduction in the immunisation calendar in 1992 [30]. People, born before 1992, however, are put at increased risk of being infected with the virus and to progress to chronic infection are still.

Further studies are needed to assess the benefits and efficiency of future implementation of national screening program for chronic viral infections. There is also a need of local epidemiology data for the progression of the disease at time of diagnosis but this could be facilitated by the implementation of such screening programme.

\section{Conclusion}

The implementation of national screening programme for $\mathrm{HCV}$ would help to find the asymptomatic patients and to ensure timely access to early treatment. This would help to reduce the chronic HCV infection-related cirrhosis, hepatocellular 
carcinoma and liver transplantation and to increase the life expectancy of the patients. The screening could increase with almost $30 \%$ the number of diagnosed people with asymptomatic chronic $\mathrm{HCV}$ infection in Bulgaria. The best possible option in terms of diagnosis is the Active Screening strategy.

\section{References}

1. Lavanchy D. Evolving epidemiology of hepatitis $C$ virus. Clin Microbiol Infect 2011; 17: 107-115.

2. Mutimer D, Aghemo A, Diepolder H, Negro F, Robaeys G, Ryder S. EASL clinical practice guidelines: management of hepatitis $\mathrm{C}$ virus infection. J Hepatol 2014; 60: 392-420.

3. Gupta E, Bajpai M, Choudhary A. Hepatitis C virus: Screening, diagnosis, and interpretation of laboratory assays. Asian J Transfus Sci 2014; 8: 19-25.

4. Gordon SC, Pockros PJ, Terrault NA, Hoop RS, Buikema A, Nerenz D, Hamzeh FM. Impact of disease severity on healthcare costs in patients with chronic hepatitis $\mathrm{C}$ (CHC) virus infection. Hepatology 2012; 56: 1651-1660.

5. Saxena V, Khungar V, Verna EC, Levitsky J, Brown RS, Hassan MA, Sulkowski MS, OLeary JG, Koraishy F, Galati JS, Kuo AA, Vainorius M, Akushevich L, Nelson DR, Fried MW, Terrault N, Reddy KR. Safety and efficacy of current direct-acting antiviral regimens in kidney and liver transplant recipients with hepatitis C: Results from the HCV-TARGET study. Hepatology 2017; 66: 1090-1101.

6. Walker DR, Pedrosa MC, Manthena SR, Patel N, Marx SE. Early view of the effectiveness of new Direct-Acting Antiviral (DAA) regimens in patients with Hepatitis C Virus (HCV). Adv Ther 2015; 32: 1117-1127.

7. Backus LI, Belperio PS. Comparative effectiveness of ledispavir/sofosbuvir +/- ribavirin vs. obmitasvir/ paritaprevir/ritonavir + dasabuvir+/- ribavirivn in 6961 genotype 1 patients treated in routine medical practice. Aliment Pharmacol Ther 2016; 44: 400-410.

8. Jacobson IM, Lawitz E, Kwo PY. Safety and efficacy of elbasavir/gazoprevir in patients with hepatitis $\mathrm{C}$ virus infection and compensated cirrhosis: an integrated analysis. Gastroenterology 2017; 152: 1372-1382.

9. Vallet-Pichard A, Pol S. Grazoprevir/elbasvir combination therapy for HCV infection. Therap Adv Gastroenterol 2017; 10: 155-167.

10. WHO Global health sector strategy on viral hepatitis 2016-2021 towards ending viral hepatitis. Geneva WHO 2016.

11. Maor Y, Malnick SD, Melzer E, Leshno M. Treatment of chronic hepatitis $\mathrm{C}$ in the aged-does it impact life expectancy? a decision analysis. PLoS One 2016; 11: 0157832.

12. Parry JV, Easterbrook $P$, Sands AR. One or two serological assay testing strategy for diagnosis of HBV and HCV infection? the use of predictive modelling. BMC Infect Dis 2017; 17: 705.
13. World Health Organization. Licence: CC BY-NC-SA 3.0 IGO 2017.

14. Pharmacotherapeutic guideline for treatment of gastrointestinal diseases/28.01.2016. Available at: http: // ncpr.bg/. Accessed on: February 2018

15. Antonov KA. Diagnostic and treatment of chronic HC infection in Bulgaria-past and present. Clujul Med 2010; 13-15.

16. Kotzev I, Ivanova I. Hepatitis C. GP News 2011; 5.

17. Okzem ZG, Akgun S. The burden of hepatitis $\mathrm{C}$ in $\mathrm{CEE}$ and CIS: An epidemiological and economic aassesment. Centre for European Policy Studies, CEPs, Bulgaria: Draft 2009.

18. Leone N, Rizzetto M. Natural history of hepatitis $\mathrm{C}$ virus infection: from chronic hepatitis to cirrhosis, to hepatocellular carcinoma. Minerva Gastroenterol Dietol 2005; 51: 31-46.

19. Clinical evidence-based guideline for breast cancer. MORE 2013. Available at: http: // www.sbaloncology.bg/bg/science-and-research/library/ more2013.html

20. Asselah T, Marcellin P. Interferon free therapy with direct acting antivirals for HCV. Liver Int 2013; 33: 93-104.

21. Coward S, Leggett L, Kaplan GG, Clement F. Costeffectiveness of screening for hepatitis $\mathrm{C}$ virus: a systematic review of economic evaluations. BMJ Open 2016; 6: 011821.

22. Ozer TT, Berktas M, Yaman G, Erkoc R. Distribution of hepatitis $\mathrm{C}$ virus genotypes in patients with chronic hepatitis C infection in Eastern Turkey. Biomed Res 2015; 26.

23. Veldt BJ, Heathcote EJ, Wedemeyer H, Reichen J, Hofmann WP, Zeuzem S, Manns MP, Hansen BE, Schalm SW, Janssen HL. Sustained virologic response and clinical outcomes in patients with chronic hepatitis $\mathrm{C}$ and advanced fibrosis. Ann Intern Med 2007; 147: 677-684.

24. van der Meer AJ, Veldt BJ, Feld JJ, Wedemeyer H, Dufour JF, Lammert F, Duarte-Rojo A, Heathcote EJ, Manns MP, Kuske L, Zeuzem S, Hofmann WP, de Knegt RJ, Hansen BE, Janssen HL. Association between sustained virological response and all-cause mortality among patients with chronic hepatitis $\mathrm{C}$ and advanced hepatic fibrosis. JAMA 2012; 308: 2584-2593.

25. Caballeria L, Pera G, Bernad J, Canut S, Navarro E, Bruguera M. Strategies for the detection of hepatitis C viral infection in the general population. Rev Clin Esp (Barc) 2014; 214: 242-246.

26. Shah HA, Heathcote J, Feld JJ. A Canadian screening program for hepatitis C: is now the time? CMAJ 2013; 185: 1325-1328.

27. Jovanovic MR, Miljatovic A, Puskas L, Kapor S, Puskas DL. Does the strategy of risk group testing for hepatitis C hit the target? Front Pharmacol 2017; 8: 437.

28. Savova A, Petrova G, Gotseva A, Kurcatova A, Koguharova M. Economic analysis 20 years after the 
introduction of universal HBV immunisation in Bulgaria.

Biotechnol Biotechnol EQ 2012; 26.

\section{*Correspondence to}

Maria Dimitrova

Medical University of Sofia

Sofia

Bulgaria 